\title{
Validation of the long-term assessment of hypothalamic-pituitary-adrenal activity in rats using hair corticosterone as a biomarker
}

\author{
Fabrizio Scorrano, ${ }^{*}{ }^{\dagger, 1}$ Javier Carrasco, ${ }^{\ddagger}, \S, \uparrow, 1$ Jordi Pastor-Ciurana, ${ }^{\ddagger}, \S, \uparrow$ Xavier Belda, ${ }^{\ddagger, \S, \uparrow}$ \\ Alicia Rami-Bastante, ${ }^{\ddagger, \S},{ }^{\uparrow}$ Maria Laura Bacci, $*$ and Antonio Armario ${ }^{\ddagger, \S, \uparrow, 2}$ \\ *Department of Veterinary Medical Sciences, Animal Physiology Unit, Faculty of Veterinary Medicine, \\ University of Bologna, 40064 Ozzano dell'Emilia, Bologna, Italy; ${ }^{\dagger}$ Unitat de Fisiologia Animal (Facultat de \\ Veterinaria), Universitat Autònoma de Barcelona, 08193 Bellaterra, Barcelona, Spain; ${ }^{\dagger}$ Unitat de \\ Fisiologia Animal (Facultat de Biociències), Universitat Autònoma de Barcelona, 08193 Bellaterra, \\ Barcelona, Spain; ${ }^{\S}$ Institut de Neurociències, Universitat Autonòma de Barcelona, 08193 Bellaterra, \\ Barcelona, Spain; and ${ }^{\top}$ Red de trastornos adictivos (RTA), Instituto de Salud Carlos III, Spain
}

ABSTRACT The evaluation of chronic activity of the hypothalamic-pituitary-adrenal (HPA) axis is critical for determining the impact of chronic stressful situations. However, current methods have important limitations. The potential use of hair glucocorticoids as a noninvasive retrospective biomarker of long-term HPA activity is gaining acceptance in humans and wild animals. However, there is no study examining hair corticosterone (HC) in laboratory animals. The present study validates a method for measuring $\mathrm{HC}$ in rats and demonstrates that it properly reflects chronic HPA activity. The HC concentration was similar in male and female rats, despite higher total plasma corticosterone levels in females, tentatively suggesting that it reflects free rather than total plasma corticosterone. Exposure of male rats to $\mathbf{2}$ different chronic stress protocols (chronic immobilization and chronic unpredictable stress) resulted in similarly higher $\mathrm{HC}$ levels compared to controls (1.8-fold). HC also increased after a mild chronic stressor (30 min daily restraint). Chronic administration of 2 different doses of a long-acting ACTH preparation dramatically increased HC (3.1- and 21.5-fold, respectively), demonstrating that a ceiling effect in $\mathrm{HC}$ accumulation is unlikely under other more natural conditions. Finally, adrenalectomy significantly reduced HC. In conclusion, HC measurement in rats appears appropriate to evaluate integrated chronic changes in circulating corticosterone. Scorrano, F., Carrasco, J., Pastor-Ciurana, J., Belda, X., Rami-Bastante, A., Bacci, M. L., and Armario, A. Validation of the long-term assessment of hypothalamicpituitary-adrenal activity in rats using hair corticosterone as a biomarker. FASEB J. 29, 000-000 (2014). www.fasebj.org

Key Words: ACTH $\cdot$ chronic immobilization $\cdot$ chronic unpredictable stress $\cdot$ glucocorticoids

Abbreviations: ADX, adrenalectomy; AUC, area under the curve; CUS, chronic unpredictable stress; HC, hair corticosterone; HPA, hypothalamic-pituitary-adrenal; IMO, immobilization; IMOch, chronic immobilization; RESTch, chronic restraint; SNK, Student-Newman-Keuls
EXPOSURE TO SYSTEMIC and emotional stressors involves a rapid activation of the hypothalamic-pituitaryadrenocortical (HPA) axis, resulting in the stimulation of adrenocortical secretion of glucocorticoids. The primary glucocorticoid in rodents is corticosterone, whereas in humans, nonhuman primates, and most mammals, it is cortisol. Corticosterone and cortisol regulate numerous biologic processes and play a critical role in the proper adaptation of the organism to stressors (1). One particularly relevant function of glucocorticoids is to inhibit the activation of the HPA axis caused by stressors through negative feedback loops exerted at different levels, including the pituitary gland, the hypothalamus, and some extrahypothalamic areas, such as the hippocampal formation and the medial prefrontal cortex (2). If exposure to the stressor is prolonged and the stress response continues, this can lead to a syndrome of distress and to deleterious effects on health (3). The activation of the HPA axis is considered a gold standard biomarker of acute stress (4), but the ability of glucocorticoids to reflect long-term HPA axis activity is limited. Thus, establishing biomarkers of chronic stress is a great challenge for investigators.

To date, glucocorticoid concentrations have been analyzed mostly in blood serum, saliva, urine, and feces, and each of these approaches has clear limitations. Glucocorticoid levels in blood and saliva reflect transient changes in the HPA axis, show high intraindividual variation, and are strongly affected by environmental disturbances (5) and circadian and ultradian rhythms (6). Currently, urine and fecal samples are widely accepted as a noninvasive technique $(7,8)$, and they reflect a somewhat longer period of HPA activity (up to 24 to $48 \mathrm{~h}$ ). However, they require repeated sampling from the same individual and are subjected to cross-contamination. More importantly, none of

\footnotetext{
${ }^{1}$ These authors contributed equally to this study.

${ }^{2}$ Correspondence: Unitat de Fisiologia Animal, Facultat de Biociències, Departament de Biología Cellular, de Fisiología i d'Immunologia, Universitat Autònoma de Barcelona, 08193 Bellaterra, Barcelona, Spain. E-mail: antonio.armario@uab.es doi: 10.1096/fj.14-254474
} 
these matrices is able to reflect the long-term accumulative impact of chronic stress exposure.

Therefore, the potential use of hair as a noninvasive, retrospective biomarker of long-term HPA activity is of great interest. Hair has been recognized as a biomaterial that accumulates glucocorticoids over weeks to months and that could eventually reflect chronic stress (9). Compared to other matrices, hair does not require special storage or shipping condition before analysis, and it is a matrix stable for years to centuries $(10,11)$. Several studies in humans have investigated the association between long-term stress exposure and hair cortisol levels, covering a wide range of physical and emotional stressors (12-17). In contrast, only 2 articles have examined the chronic hair cortisol response to chronic stress in rhesus macaques and vervet monkeys $(9,18)$.

It has been assumed that hair glucocorticoids reflect free rather than total plasma glucocorticoids $(19,20)$, but evidence is scarce. In rats, total plasma corticosterone levels are clearly higher in female compared to male rats $(21,22)$, but plasma levels of corticosteroid-binding globulin are also higher in females (23). Hence, it appears that the free fraction of circulating corticosterone is similar in male and female rats, as supported by similar brain levels of corticosterone $(24,25)$. Similar levels of hair corticosterone (HC) in both sexes could provide evidence for the latter possibility.

To our knowledge, there is no report on the technical validation of the measurement of glucocorticoids (corticosterone) in the hair of laboratory rodents and its utility to evaluate chronic stress. This is critical because prior studies in humans and nonhuman primates, although of great interest, offer few opportunities to introduce manipulations that could more appropriately validate the use of hair as a measure of chronic HPA activation. For instance, it is not known whether hair glucocorticoids reflect the intensity of the chronic stressful situation and the amount of glucocorticoid released. Moreover, this evaluation is needed considering the extensive use of rodents in animal models of diseases. Thus, the aims of the present work were to develop and validate a reliable noninvasive method for measuring $\mathrm{HC}$ concentration in rats; to provide evidence that supports the hypothesis that hair glucocorticoids is representative of the free rather than the total fraction of circulating glucocorticoids; and to demonstrate that $\mathrm{HC}$ accurately reflects long-term HPA axis activity.

\section{MATERIALS AND METHODS}

\section{Animals and general procedures}

Male and female Sprague Dawley rats obtained from the breeding center of the Universitat Autònoma de Barcelona were used. Animals were approximately $60 \mathrm{~d}$ old at the beginning of the experiments and were assigned randomly to the experimental groups. Animals were housed individually (experiments 1 and 2) or in pairs (experiment 3 ) in polypropylene opaque wire-topped cages with a solid bottom $\left(21.5 \times 46.5 \times 14.5 \mathrm{~cm}\right.$; Type " $1000 \mathrm{~cm}^{2}$ ", Panlab SLU, Barcelona, Spain) containing wood-shaving bedding (Lignocel 3/4; Harlan Interfauna Ibérica, Barcelona, Spain); standard temperature conditions $\left(21 \pm 1^{\circ} \mathrm{C}\right)$ and a $12-12 \mathrm{~h}$ light/dark schedule (lights on at 8:00 AM) were provided. Food (Global Diet 2014, Harlan Laboratories, Madison, WI) and water were available ad libitum. Animals were individually housed in some experiments because they took part in a larger experiment studying the influence of stress on saccharine intake (26), which required individual housing. We have previously reported that there are no major differences in general parameters and HPA function in individually housed versus group-housed male Sprague Dawley rats (27). The animals were allowed to acclimate to the housing conditions for at least 1 week before beginning the experimental treatments. If not otherwise stated, experiments were carried out in the morning. The experimental protocols were approved by the ethics committee of the Universitat Autònoma de Barcelona following established principles of laboratory animal care, and they were carried out in accordance to the European Communities Council Directive (2010/63/EU).

Starting at least $3 \mathrm{~d}$ after their arrival, all animals were handled 3 times ( 2 min each) over a period of 1 week. The experiments started 48 to $72 \mathrm{~h}$ after the third handling session. On the last day of handling, animals were subjected to a tail-nick procedure to habituate them to the blood sampling procedure. The tail-nick consisted of gently wrapping the animals with a cloth, making a $2 \mathrm{~mm}$ incision at the end of one of the tail veins, and then massaging the tail while collecting, within $2 \mathrm{~min}, 300 \mu \mathrm{l}$ of blood into ice-cold EDTA capillary tubes (Sarstedt, Granollers, Spain). The tail-nick procedure is extensively used in our laboratory because low resting levels of hormones are obtained (28). After centrifugation $\left(4960 \times g, 15 \mathrm{~min}\right.$ at $\left.4^{\circ} \mathrm{C}\right)$, plasma was stored at $-20^{\circ} \mathrm{C}$. In all experiments, cage mates were processed simultaneously, including blood sampling. Animals were always stressed in a room different from the animal room and the sampling room.

Only a few seconds of wrapping the animals was necessary to collect a large amount of hair. Given its lipophilic nature, corticosterone is assumed to be incorporated into follicles during the anagen phase of the hair through passive diffusion from the blood (29-31). Thus, hair was shaved in the morning with an electric grooming clipper as close to the skin as possible. Approximately $50 \mathrm{~cm}^{2}$ of old hair was removed from the region delimited cranially by the eighth lumbar vertebra, caudally by the ischial tuberosity, and laterally by the posterior limbs. This area corresponds approximately to the ischiatic region of the animal. If a second sample of new hair was required by the experimental design, the sample was collected within the same region, with the technician being careful not to cut any old hair. Samples were placed into aluminum foil for protection, then into small plastic pouches. These were numbered and kept at $4^{\circ} \mathrm{C}$ until the time of assay.

\section{Experiment 1: comparison of male and female rats}

Sixteen rats ( 8 male and 8 female) were housed individually and blood samples were taken under basal conditions at different time points on a single day (10:00 AM, 1:00 PM, 5:00 PM, 8:00 PM, 11:00 PM, 10:00 AM) to assess total plasma corticosterone circadian rhythm. Hair samples were collected at the end of the experiment.

\section{Experiment 2: effect of 2 chronic stress procedures on HC}

Experiment 2 was conducted to assess if 2 relatively severe chronic stress models increase HC. Eighteen male rats housed individually were randomly assigned to the following 3 groups: control $(n=6)$, chronic immobilization (IMOch) $(n=6)$, and chronic unpredictable stress (CUS) $(n=6)$. On d 1 before starting the treatments, hair samples were taken from all animals. Body weight and food intake were measured several times throughout the experimental period. Hair was sampled at d 12 instead of on the day after the last stressor (d 10) because hair growth was not sufficient for removal. Animals were then humanely killed, and their adrenal gland and thymus were removed. 
Two chronic stress procedures were IMOch and CUS. The chronic treatment lasted from experimental d 1 to 9 . Control animals remained undisturbed. The first model consisted of daily exposure to $1 \mathrm{~h}$ of immobilization (IMO) at 9:30 AM. IMO rats were stressed by taping their 4 limbs to metal mounts attached to a board (32). Head movements were restricted with 2 plastic pieces $(7 \times 6 \mathrm{~cm})$ placed in each side of the head and the body was subjected to the board by means of a piece of plastic cloth (10 cm wide) attached with Velcro that surrounded the trunk. The CUS procedure (26) consisted of exposing rats to different types of stressors: restraint, electric foot shocks, and forced swimming. For restraint, animals were placed into PVC cylindrical tubes measuring $6 \mathrm{~cm}$ in diameter and $21.5 \mathrm{~cm}$ in length for 30 , 60 , or $90 \mathrm{~min}$. The rear top of the apparatus was closed by a cork, letting the tail of the rat hang off. Several holes $(0.5 \mathrm{~cm}$ in diameter) in the walls of the cylinder provided fresh air. For shock, rats received a $6 \mathrm{~s}$ shock $(1.5 \mathrm{~mA})$ each minute for 30,60 , or $90 \mathrm{~min}$. Rats were put into individual clear Plexiglas boxes with a metal grid floor connected to a shocker (Cibertec, Madrid, Spain). For the swimming condition, animals were placed in transparent cylindrical plastic tanks (height $40 \mathrm{~cm}$, internal diameter $19 \mathrm{~cm})$ containing water $\left(25^{\circ} \mathrm{C}\right)$ to a level of $24 \mathrm{~cm}$, as described previously (33). Animals remained in the tank for $20 \mathrm{~min}$. The rats were then withdrawn from the water, returned to their home cages, and exposed to swim for an additional $10 \mathrm{~min}$ period $1 \mathrm{~h}$ later. Similar to other well-established chronic variable stress procedures (34), our CUS model involved changing the stressor and the time every day: $\mathrm{d}$ 1, $30 \mathrm{~min}$ restraint at 11:00 AM; d 2, 30 min of shocks at 12:30 PM; d 3, swim at 3:00 PM; d 4, 60 min of shocks at 10:30 AM; d 5, swim at 8:00 AM; d 6, 60 min of restraint at 3:30 PM; $d$ 7, swim at 4:00 PM; d 8, 90 min of restraint at 10:00 AM; and d 9, 90 min of shocks at 2:30 PM.

\section{Experiment 3: effects of an intermediate-intensity chronic stressor (restraint)}

Experiment 3 was conducted to assess if daily repeated exposure to an intermediate-intensity, extensively used stressor (restraint) could increase HC. We have previously observed that restraint is of lower intensity than IMO (35). Eighteen male rats housed individually were randomly assigned to control $(n=8)$ or chronic restraint stress (RESTch) $(n=8)$ groups. The latter animals were exposed daily to $30 \mathrm{~min}$ of restraint for $14 \mathrm{~d}$. The restraint was carried out in PVC cylindrical tubes measuring $6 \mathrm{~cm}$ in diameter and $21.5 \mathrm{~cm}$ in length. The rear top of the apparatus was closed by a cork, letting the tail of the rat hang off. Several holes $(0.5 \mathrm{~cm}$ in diameter) in the walls of the cylinder provided fresh air. On the day before starting stress exposure, hair samples were taken from all animals and again on d 15. Body weight and food intake were measured several times throughout the experimental period. Animals were then humanely killed, and their adrenal gland and thymus were removed.

\section{Experiment 4: effect of chronic ACTH administration}

The aim of experiment 4 was 2-fold: first, to demonstrate that HC quantitatively reflects the magnitude of chronic corticosterone production, and second, to rule out a ceiling effect in hair accumulation that limits its validity to reflect actual corticosterone secretion under certain conditions. To this end, we administered tetracosactide (Nuvacthén Depot $1 \mathrm{mg} / \mathrm{ml}$; Defiante farmacêutica, Funchal, Portugal), a synthetic analog of ACTH consisting of the first 24 amino acids of the naturally occurring hormone. This treatment maintains high levels of ACTH over the $24 \mathrm{~h}$ period, thus resulting in a marked and prolonged activation of adrenocortical secretion (36) while maintaining physiologic levels of glucocorticoids.
Twenty-four male rats housed in pairs were randomly assigned to 3 groups (8 rats per group) and received daily saline solution (control) or tetracosactide (20 or $100 \mu \mathrm{g} / \mathrm{kg}$ ) for $15 \mathrm{~d}$ to allow appropriate hair growth. Injections were administered subcutaneously from d 1 to 15 at 10:00 AM. Hair samples were collected on d 1 (before starting the treatments) and again on $\mathrm{d} 16$. Body weight and food intake were monitored throughout the experimental period. The values reported are the food consumption per $d$ per rat, and the statistical unit was the cage. At the end of the experiment, animals were humanely killed, and the adrenal gland removed.

\section{Experiment 5: effect of adrenalectomy}

Experiment 5 was conducted to assess if $\mathrm{HC}$ quantitatively reflects the strong reduction of circulating corticosterone after adrenalectomy (ADX). Eight male rats housed individually were randomly assigned to ADX- and sham-treated groups $(n=4$ per group). All animals were weighed and shaved on the day before surgery, and again on d 15 and 30 after surgery. Two post-ADX periods were studied because a prior pilot study demonstrated only partial decrease of $\mathrm{HC}$ in the 2 weeks after ADX. Blood samples were collected at $\mathrm{d} 14$ and 29 at 2 time points on a single day (10:00 AM and 7:00 PM), as described above. ADX was performed in rats anesthetized with $2 \%$ to $3 \%$ isoflurane (IsoFlo Fl. $250 \mathrm{ml}$; Esteve Spa, Barcelona, Spain) and placed in ventral recumbency. A dorsal midline incision was made with its midpoint centered over the 13th rib, and the underlying muscle wall on either side of the spinal column was pierced by scissors. The adrenal glands were located, and the forceps were used to tear away the gland and its fat pad. The abdominal muscle was sutured with single interrupted absorbable suture, and the skin incision was closed with 1 to 2 simple interrupted nonabsorbable sutures. The sham-treated group was treated the same way as ADX animals but the adrenal glands were not removed. Buprenorphine (Buprex $0.3 \mathrm{mg} / \mathrm{ml}$; RB Pharmaceuticals Limited, Barcelona, Spain) was injected subcutaneously $30 \mathrm{~min}$ before surgery and after $24 \mathrm{~h}$ to all animals. ADX rats received $0.9 \%$ saline as drinking solution after surgery until the end of the experiment. Animals were then humanely killed, and necropsy was performed to ensure the absence of adrenal tissue.

\section{Corticosterone radioimmunoassay and validation of the $\mathrm{HC}$ assay method}

Plasma and HC levels were assayed by double antibody radioimmunoassay. In brief, corticosterone radioimmunoassay used $\left[{ }^{125} \mathrm{I}\right]$ corticosterone-carboxymethyloxime-tyrosine-methylester (ICN-Laboratorios Leti, Barcelona, Spain) and synthetic corticosterone (Sigma, Barcelona, Spain) as the standard and an antibody raised in rabbits against corticosterone-carboximethyloximebovine serum albumin that was kindly provided by Dr. G. Makara (Institute of Experimental Medicine, Budapest, Hungary). The characteristics of the antibody and the basic radioimmunoassay procedure have been described previously (37). The range of the standard curve was between $6.25 \mathrm{pg}$ and $1600 \mathrm{pg}$ of corticosterone per tube. The antibody used has a cross-reactive of $2.3 \%$ with progesterone, $1.5 \%$ with desoxycorticosterone, and less than $0.1 \%$ with any other steroid tested. All samples that were statistically compared were run in the same assay to avoid interassay variability.

For HC extraction, we developed a washing protocol based on that reported by Davenport et al. (9) in rhesus macaques and subsequently applied successfully in other species $(38,39)$. For corticosterone extraction, hair samples were washed twice by mixing with $15 \mathrm{ml}$ of undiluted 2-propanol (Sigma, Barcelona, Spain) per $\sim 200 \mathrm{mg}$ of hair using a tube mixer that provides $360^{\circ}$ rotation at $20 \mathrm{rpm} / \mathrm{min}$ (Minilabroller; Sigma, Barcelona, Spain); 
each wash was followed by centrifugation at $1500 \times g\left(1 \mathrm{~min}, 4^{\circ} \mathrm{C}\right)$ and removal of the supernatant. Hair samples were put on aluminum foil inside an incubator at $37^{\circ} \mathrm{C}$ for approximately $30 \mathrm{~min}$ to completely dry the samples. Dried hair was frozen with liquid nitrogen and minced in a mortar while it was frozen. Five milliliters of HPLC-grade methanol (Scharlau, Barcelona, Spain) was added to $100 \mathrm{mg}$ of powdered hair and mixed at room temperature by rotation at $20 \mathrm{rpm} / \mathrm{min}$ for $21 \mathrm{~h}$. After a centrifugation at $10,000 \times g\left(10 \mathrm{~min}, 4^{\circ} \mathrm{C}\right), 4 \mathrm{ml}$ of supernatant was recovered in a glass tube and dried at $38^{\circ} \mathrm{C}$ under a gentle stream of nitrogen gas. Dried extracts were reconstituted with $200 \mu \mathrm{l}$ of $0.2 \mathrm{M}$ sodium phosphate buffer, $\mathrm{pH} 7.6$, and stored at $-20^{\circ} \mathrm{C}$. On the day of analysis, samples were centrifuged at $16,000 \times g\left(30 \mathrm{~min}, 4^{\circ} \mathrm{C}\right)$, and the supernatant was recovered and assayed. In the final validated protocol, $10 \mu \mathrm{l}$ of reconstituted hair extract was used per tube.

\section{Statistical analysis}

Data were analyzed by SPSS software, version 17 (IBM, Armonk, NY). A 2-way ANOVA with sex as the between-subjects factor (2 levels) and time of day as the within-subjects factor (6 levels) was used for the study of the circadian rhythm of plasma corticosterone in Experiment 1. When appropriate, further comparisons were performed. In other cases, a $t$ test was used to compare 2 independent means (e.g., sex differences in HC). The 1-way ANOVA was followed by a post hoc Student-Newman-Keuls (SNK) test when more than 2 independent means were compared (the criterion for significance was set at $P<0.05$ in the SNK test). When variances were not homogenous between groups, logarithmic transformation of data was used. The area under the curve (AUC) for each animal was calculated from the circadian rhythm data with GraphPad Prism 5. To calculate AUC, corticosterone concentration (nanograms per milliliter) was plotted in the $y$ axis $v s$. time $(h)$ in the $x$ axis. The area is computed connecting a straight line between every set of adjacent points defining the curve; it sums up the area beneath these lines.

\section{RESULTS}

\section{Technical validation}

To validate the described methods, the linearity, accuracy, precision, and reproducibility of the assay were studied (Tables 1 and 2). Assay linearity was evaluated by assaying 3 different dilutions of 3 independent samples. A good parallelism between sample dilution and standard curve was found. To evaluate assay accuracy, each of 5 reconstituted

TABLE 1. Linearity

\begin{tabular}{lccr}
\hline \hline Sample & Hair used $(\mathrm{mg} /$ tube $)$ & Corticosterone $(\mathrm{pg} / \mathrm{mg})$ & $\%$ \\
\hline 1 & 8 & $19.9 \pm 0.3$ & 100 \\
& 4 & $20.5 \pm 0.1$ & 103 \\
& 2 & $20.6 \pm 0.5$ & 104 \\
& 1.4 & $14.8 \pm 0.2$ & 100 \\
& 0.7 & $14.4 \pm 0.5$ & 97 \\
3 & 0.35 & $15.1 \pm 0.3$ & 102 \\
& 8 & $16.6 \pm 0.6$ & 100 \\
& 4 & $20.4 \pm 0.5$ & 123 \\
& 2 & $22.8 \pm 0.8$ & 137 \\
\hline
\end{tabular}

To assay linearity, 3 independent samples were diluted at a ratio of 1:2 and 1:4. The average concentration (mean \pm SEM) and the percentage of the expected value for each sample are shown.
TABLE 2. Recovery

\begin{tabular}{|c|c|c|c|}
\hline \multirow[b]{2}{*}{ Sample } & \multicolumn{2}{|c|}{ Corticosterone } & \multirow[b]{2}{*}{$\%$} \\
\hline & Added (pg/tube) & Observed (pg/tube) & \\
\hline \multirow{5}{*}{4} & 0 & $74.1 \pm 4.8$ & \\
\hline & 50 & $133.4 \pm 15.8$ & 107 \\
\hline & 100 & $168.4 \pm 8.0$ & 97 \\
\hline & 200 & $273.0 \pm 0.4$ & 100 \\
\hline & 0 & $39.2 \pm 3.5$ & \\
\hline \multirow{3}{*}{5} & 50 & $97.8 \pm 0.8$ & 110 \\
\hline & 100 & $152.7 \pm 4.6$ & 110 \\
\hline & 200 & $222.9 \pm 18.0$ & 93 \\
\hline \multirow{5}{*}{6} & 0 & $13.8 \pm 1.7$ & \\
\hline & 50 & $52.5 \pm 4.6$ & 81 \\
\hline & 100 & $114.0 \pm 19.8$ & 100 \\
\hline & 200 & $188.4 \pm 24.0$ & 88 \\
\hline & 0 & $40.8 \pm 4.9$ & \\
\hline \multirow{3}{*}{7} & 50 & $73.0 \pm 0.2$ & 80 \\
\hline & 100 & $143.5 \pm 2.9$ & 102 \\
\hline & 200 & $235.8 \pm 26.0$ & 98 \\
\hline \multirow{4}{*}{8} & 0 & $14.9 \pm 0.2$ & \\
\hline & 50 & $73.6 \pm 2.3$ & 114 \\
\hline & 100 & $101.5 \pm 0.2$ & 89 \\
\hline & 200 & $216.5 \pm 8.2$ & 100 \\
\hline
\end{tabular}

To assay recovery, 3 known concentrations of corticosterone were added ( $+50 \mathrm{pg} ;+100 \mathrm{pg}$ and $+200 \mathrm{pg}$ per tube) to 5 hair extracts. The average concentration (mean \pm SEM) is shown. Percentage recovery was calculated on observed vs. expected.

hair extracts were divided in 3 independent aliquots and spiked with 3 different known corticosterone concentrations, mixed, and assayed. The percentage of recovery was determined as follows: [ (measured corticosterone in spiked sample)/(measured corticosterone in nonspiked sample + corticosterone added) $\times 100]$. Recovery was $97.9 \pm 10.3 \%$ (mean $\pm \mathrm{SD}$ ), indicating a good selectivity of the assay. Taken together, parallelism and selectivity results demonstrate the proportionality between $\mathrm{HC}$ and the reference standard and the absence of significant matrix interferences, at least in the range of hair extracts assayed. Precision was estimated by repeatedly extracting and assaying samples in the same assay (6 times). Reproducibility was estimated by assaying 3 samples in 8 independent assays. The mean intra-assay and interassay coefficient of variation were $4.3 \%$ and $12.2 \%$, respectively. The lowest corticosterone standard used in the assay was $6.25 \mathrm{pg}$ per tube; using $8 \mathrm{mg}$ of hair per tube, $\mathrm{HC}$ can be assayed with reasonable accuracy and precision with a sensitivity of $0.78 \mathrm{pg} / \mathrm{mg}$ hair.

\section{Biologic validation \\ Comparison of male and female rats}

Figure 1 shows a comparison of corticosterone concentration in male and female rats. The 2-way ANOVA of the circadian pattern of plasma corticosterone revealed significant effects of sampling time $(F(5,70)=4.6, P<0.001)$, sex $(F(1,14)=7.1, P=0.019)$ and the interaction sampling time $\times \operatorname{sex}(F(5,70)=3.9, P=0.021)$. The decomposition of the interaction showed that plasma corticosterone levels were higher in females at 10:00 AM. The $t$ test analysis revealed that the corticosterone AUC was significantly 

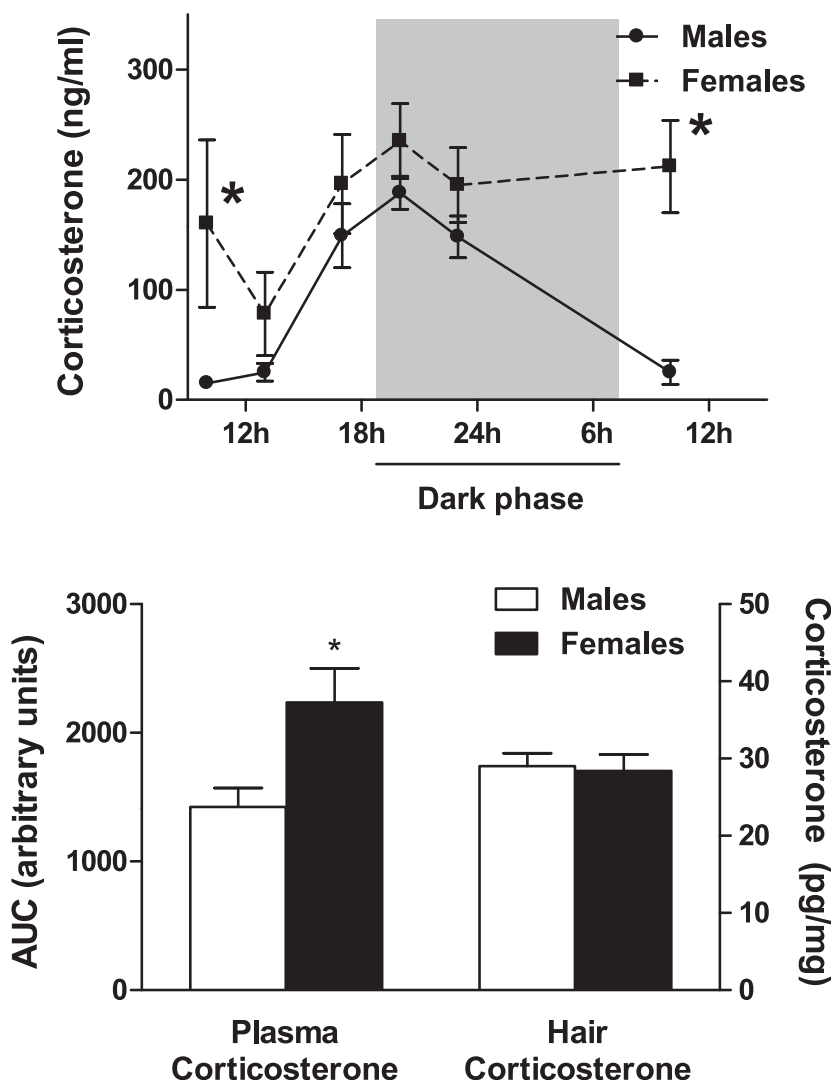

Figure 1. Plasma and HC levels in male and female rats. Top, circadian rhythm of total plasma corticosterone. Samples were taken at different time points during the lights-on period (10:00 AM, 1:00 PM, and 5:00 PM) and the nocturnal period (8:00 PM and 11:00 PM, marked in shadow). Means \pm SEM $(n=$ 8 per group) are presented. Circadian rhythm data were analyzed by 2-way ANOVA with repeated measures for the factor time. AUC of plasma corticosterone was calculated from the circadian rhythm data for each animal (bottom left). $\mathrm{HC}$ levels are shown at bottom right; $* P<0.05$ between sexes.

higher in females than males $(t(14)=2.68, P=0.018)$. However, no differences were found in the HC concentration between sexes $(t(14)=-0.22, P=0.826)$.

\section{Effects of 2 chronic severe stressors}

Table 3 shows the results for body weight gain, food intake, and adrenal and thymus weights. Statistical analysis revealed significant differences between groups in body weight gain $(F(2,17)=26.2, P<0.001)$ and food intake $(F(2,17)=14.3, P<0.001)$. Post hoc comparisons with the SNK test showed that CUS decreased body weight gain compared to controls, but the effect of IMOch was more pronounced and differed from both control and CUS rats. Although CUS tended to decrease food intake, the effect only reached significance in IMOch compared to controls. Neither adrenal nor thymus weight were significantly affected by CUS or IMOch.

The amount of hair obtained after chronic exposure to the stressors was similar in all groups, suggesting no effect of stress on hair growth (data not shown). HC concentrations after the chronic stress procedures are shown in
Fig. 2. We separately analyzed HC before and after chronic stress because they represent completely different time domains for corticosterone accumulation, and hair growth is not uniform across its cycle (40). Samples taken after chronic stress represents only the period of exposure to chronic stress. As expected, the 1-way ANOVA revealed no group differences on $\mathrm{d} 1$ samples (before chronic stress) (control, $16.5 \pm 1.6 \mathrm{pg} / \mathrm{mg}$; IMOch, $16.2 \pm 1.8 \mathrm{pg} / \mathrm{mg}$; CUS, $17.1 \pm 1.6 \mathrm{pg} / \mathrm{mg}$ ). The 1-way ANOVA revealed significant group differences on d $12(F(2,17)=10.2, P=$ 0.002). Further post hoc comparisons showed that both IMOch and CUS significantly increased $\mathrm{HC}$ concentration (approximately 80\%) compared to controls $(P<0.05)$, with no differences between both chronic stress paradigms.

\section{Effects of RESTch}

RESTch did not alter food intake or adrenal and thymus weights (data not shown) but significantly reduced body weight gain (90.8 g vs. $69.0 \mathrm{~g}, t(14)=4.73, P<0.001)$. Regarding $\mathrm{HC}$, no differences between groups were observed on d 1 (before RESTch) (control, $31.0 \pm 4.1 \mathrm{pg} / \mathrm{mg}$; RESTch, $35.9 \pm 2.7 \mathrm{pg} / \mathrm{mg}$ ). Because we can predict that chronic stress should increase HC, the 1-tailed $t$ test was used that revealed significant increase after the chronic stress procedure $(t(14)=-1.82, P=0.045)($ Fig. 3) .

\section{Effects of chronic ACTH administration}

Table 4 shows the results for body weight gain, food intake, and adrenal weight. The 1-way ANOVA revealed significant differences between groups in absolute body weight $(F(2,23)=17.42, P<0.001)$, body weight gain $(F(2,23)=$ $70.4, P<0.001$ ), both absolute and relative adrenal weights $(F(2,23)=93.7$ and $F(2,23)=110.0$, respectively, $P<0.001$ in both cases), and food intake $(F(2,11)=6.3, P=0.019)$. Post hoc SNK comparisons showed that only the higher dose of ACTH significantly decreased absolute body weight, but both doses of ACTH caused a statistically significant reduction in body weight gain, with a significantly greater effect in the high-dose group. These results illustrate that greater sensitivity of body weight gain instead of absolute body weight to detect the impact of stress. Changes in absolute and relative adrenal weight followed the same pattern as body weight gain. ACTH treatment caused a reduction in food intake that was statistically significant only for the high dose.

Regarding HC, the 1-way ANOVA did not reveal significant differences between groups on $\mathrm{d} 1$ (before drug administration) (control, $25.6 \pm 1.3 \mathrm{pg} / \mathrm{mg} ; 20 \mu \mathrm{g} / \mathrm{kg}$, $27.5 \pm 1.8 \mathrm{pg} / \mathrm{mg} ; 100 \mu \mathrm{g} / \mathrm{kg}, 27.0 \pm 1.8 \mathrm{pg} / \mathrm{mg})$ but did on d $16(F(2,23)=182.7, P<0.001)$ (Fig. 4). A post hoc SNK test revealed that both doses dramatically increased $\mathrm{HC}$ concentration $(P<0.05)$, with a much greater increase in the $100 \mu \mathrm{g} / \mathrm{kg}$ group. In the latter case, ACTH administration caused more than a 20-fold increase in HC levels.

\section{Effect of $A D X$}

ADX greatly decreased the circulating corticosterone levels to less than $10 \mathrm{ng} / \mathrm{ml}$, even in the peak of the circadian 


\begin{tabular}{|c|c|c|c|c|c|c|c|c|}
\hline \multirow[b]{2}{*}{ Group } & \multirow[b]{2}{*}{$\begin{array}{l}\text { Body weight } \\
\text { at d } 9(\mathrm{~g})\end{array}$} & \multirow[b]{2}{*}{$\begin{array}{l}\text { Body weight } \\
\text { gain }(\mathrm{g})\end{array}$} & \multirow[b]{2}{*}{$\begin{array}{c}\text { Food intake } \\
(\mathrm{g} / \mathrm{rat} / \mathrm{d})\end{array}$} & \multirow[b]{2}{*}{$\begin{array}{c}\text { Body weight at } \\
\text { d } 12(\mathrm{~g})\end{array}$} & \multicolumn{2}{|c|}{ Adrenal weight } & \multicolumn{2}{|c|}{ Thymus weight } \\
\hline & & & & & Absolute (mg) & $\begin{array}{c}\text { Relative } \mathrm{mg} \text { of } \\
\text { (tissue/ } 100 \mathrm{~g} \\
\text { body weight) }\end{array}$ & Absolute (mg) & $\begin{array}{r}\text { Relative mg of } \\
\text { (tissue } / 100 \mathrm{~g} \\
\text { body weight) }\end{array}$ \\
\hline Control & $375.7 \pm 17.8$ & $60.5 \pm 2.5$ & $28.2 \pm 1.0$ & $441.4+$ & $57.2 \pm 3.1$ & $13.0 \pm 0.7$ & $576.9 \pm 65.1$ & $129.3 \pm 10.6$ \\
\hline IMOch & $329.0 \pm 14.9$ & $12.2 \pm 5.7 *$ & $22.2 \pm 0.8^{*}$ & $395.0 \pm 29.0$ & $53.8 \pm 2.5$ & $13.6 \pm 0.6$ & $450.3 \pm 52.7$ & $115.9 \pm 16.0$ \\
\hline CUS & $352.9 \pm 12.1$ & $29.9 \pm 5.4^{* \$}$ & $26.2 \pm 0.5$ & $389.6 \pm 29.8$ & $59.6 \pm 3.1$ & $15.3 \pm 0.7$ & $451.8 \pm 33.1$ & $117.2 \pm 10.7$ \\
\hline
\end{tabular}

Total body weight gain and average food intake per day during the chronic stress period (between $\mathrm{d} 1$ and 9 ). Adrenal and thymus weights were measured at euthanasia on d 12. Means \pm SEM ( $n=8$ per group) are presented. Group differences were analyzed by a 1-way ANOVA followed by SNK post hoc comparisons; ${ }^{*} P<0.05$ vs. control group; ${ }^{\$} P<0.05$ between IMOch (chronic immobilization) and CUS (chronic unpredictable stress) groups.

cycle. There were no differences between groups on HC on d 1 (sham treated, $17.8 \pm 1.5 \mathrm{pg} / \mathrm{mg}$; ADX treated, $17.4 \pm 1.8 \mathrm{pg} / \mathrm{mg})$. However, a progressive decrease in HC-treated compared to the sham-treated group was found on d 15 and 30 after surgery (Fig. 5). The $t$ test revealed statistically significant differences between ADX-and sham-treated rats on both on d $15(t(6)=3.32$, $P=0.016)$ and $\mathrm{d} 30(t(6)=10.08, P<0.001)$.

\section{DISCUSSION}

The present results represent what is to our knowledge the first technical and biologic validation of $\mathrm{HC}$ measurement in rats. We have validated a specific washing protocol and extraction assay method that has good sensitivity. More importantly, the present results provide tentative support to the hypothesis that $\mathrm{HC}$ reflects plasma-free corticosterone fraction and that it is a good marker of chronic HPA activation in typical chronic stress procedures lasting several weeks.

The results of the first experiment showed the expected and well-characterized circadian pattern of plasma corticosterone with lower levels in the morning and the highest levels occurring around the onset of the dark cycle. As expected $(21,22)$, females showed higher levels of

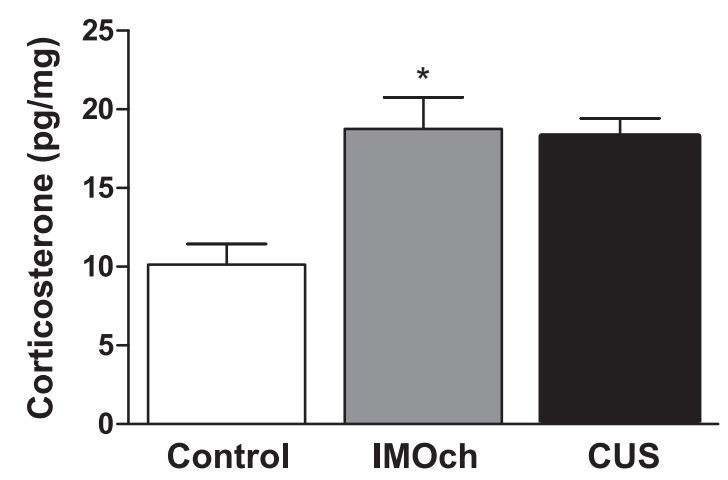

Figure 2. Effects of 2 chronic stress procedures on HC. Chronic exposure to IMO (IMOch) or to various different stressors (CUS) started on d 1 and was completed on d 9. Hair samples were taken before the experiment (d 1) and at $\mathrm{d} 12$. Only the results for $\mathrm{d} 12$ are shown. Means $\pm \operatorname{SEM}(n=6$ per group) are presented. Groups were statistically compared by 1-way ANOVA followed by SNK post hoc test when appropriate; $* P<0.05$ vs. control group. corticosterone throughout the $24 \mathrm{~h}$ period than males. However, HC concentration was similar in both sexes. Because it has been reported that brain corticosterone concentration, which reflects free plasma corticosterone, is similar in male and female rats $(24,25)$, our results support the hypothesis $(19,20)$ that hair glucocorticoids are a reflection of free rather than total circulating glucocorticoid levels. The present results are supported by prior studies in humans and other species that failed to find sex differences in hair cortisol or higher levels in men under nonstressful conditions $(14,15,29,41-43)$. Small and inconsistent differences have been reported in salivary cortisol, which reflects free cortisol, between men and women under resting conditions (44-46). However, women consistently respond less to acute stressors (47). Nevertheless, alternative possibilities cannot be completely ruled out, including sex differences in the rate of hair growth or HC accumulation/degradation that may compensate for differences in circulating corticosterone. Moreover, sex differences could appear after exposure to chronic stress rather than under basal conditions.

Hair cortisol has attracted great interest as a means to evaluate chronic stress in humans $(20,43,48)$ and has potential as a fundamental tool in human stress research. However, studies in experimental animals are needed to validate the procedure. To our knowledge, there are only 2 studies that aimed to characterize hair cortisol changes after chronic stress in nonhuman primates. Chronic stress

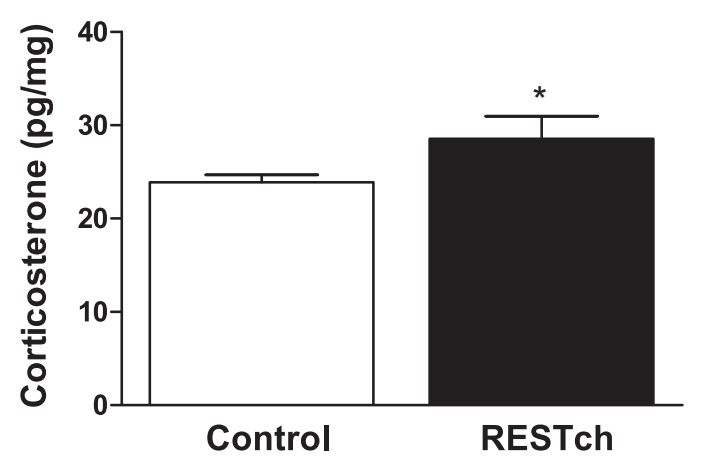

Figure 3. Effects of RESTch on HC. Animals were exposed to RESTch for $14 \mathrm{~d}$. Hair samples were taken before the experiment $(\mathrm{d} 1)$ and at $\mathrm{d} 15$. Only the results for $\mathrm{d} 15$ are shown. Means \pm SEM $(n=8$ per group) are presented. Groups were statistically compared by $t$ test; $* P<0.05$ vs. control group. 


\begin{tabular}{llcccc}
\hline \hline & & & & Adrenal weight \\
\cline { 5 - 6 } \multicolumn{1}{c}{ Dose } & Body weight $(\mathrm{g})$ & Body weight gain $(\mathrm{g})$ & Food intake $(\mathrm{g} / \mathrm{rat} / \mathrm{d})$ & Absolute $(\mathrm{mg})$ & Relative (mg of tissue/100 g body weight) \\
\hline Vehicle & $344,9 \pm 9.0$ & $84.3 \pm 4.3$ & $47.0 \pm 1.7$ & $41.5 \pm 2.3$ & $12.0 \pm 0.5$ \\
$20 \mu \mathrm{g} / \mathrm{kg}$ & $324,4 \pm 8.9$ & $63.6 \pm 3.5^{*}$ & $42.4 \pm 1.0$ & $56.5 \pm 4.0^{*}$ & $17.5 \pm 1.3^{*}$ \\
$100 \mu \mathrm{g} / \mathrm{kg}$ & $273,7 \pm 8.3^{*}$ & $12.8 \pm 5.2^{*}$ & $39.1 \pm 1.9^{*}$ & $113.9 \pm 5.0^{*}$ & $42.0 \pm 2.4^{*}$ \\
\hline
\end{tabular}

Total body weight gain ( $n=8$ per group) and average food intake per day and cage ( $n=4$ per group) during the chronic administration period (between $\mathrm{d} 1$ and 15). Absolute body weight and adrenal weight were measured on $\mathrm{d} 16$. In this experiment, animals were housed in pairs, so food consumption refers to the home cage intake, not each individual animal's intake. Means \pm sEm are presented. Group differences were analyzed by 1-way ANOVA followed by SNK post hoc comparisons; ${ }^{*} P<0.05$ vs. vehicle group; ${ }^{\$} P<0.05$ vs. lower dose of drug.

was induced by moving animals from one facility to another or to a new social environment; in both cases, higher hair cortisol levels were observed 14 and $29 \mathrm{wk}$ after the chronic stress $(9,18)$.

In the present experiment, we directly assessed the influence of 2 chronic stress models: chronic repeated IMO and CUS. By measuring not only HC but also other wellknown stress-related parameters (food intake, body weight gain, adrenal and thymus weight), we can better characterize the overall intensity of the stressors and compare the above parameters with the changes in HC concentration. IMOch and CUS both significantly reduced body weight gain, and the former also reduced food intake, suggesting that a greater overall physiologic impact of IMOch than CUS. However, both stressors increased HC concentration to the same extent, demonstrating for the first time that $\mathrm{HC}$ is appropriate to evaluate chronic stress in laboratory rodents. A contribution of chronic stress-induced changes in hair growth was ruled out because no group differences have been observed. Although food intake and body weight gain are rather good markers of the severity of stressors, a partial dissociation between different stress markers is not unlikely. Interestingly, in the present experiment, changes in relative adrenal weight of the adrenals and thymus were not significant. Thus, it appears that the measurement of $\mathrm{HC}$ appropriately reflects chronic

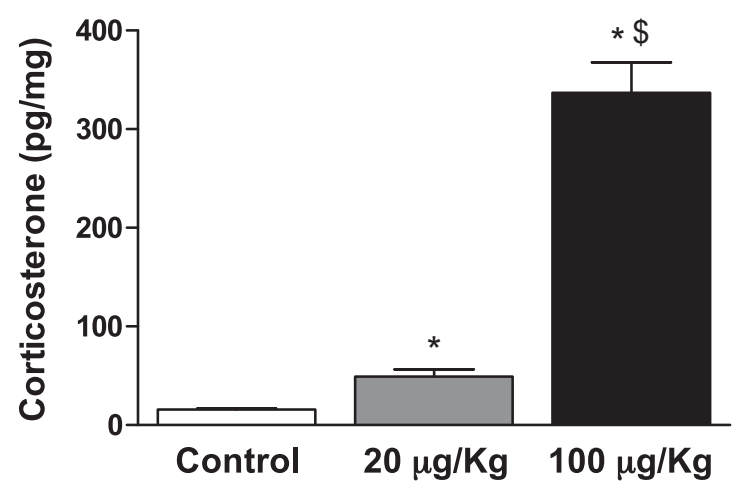

Figure 4. Effects of chronic ACTH (tetracosactide) administration on HC. Vehicle or 2 doses of tetracosactide $(20 \mu \mathrm{g} / \mathrm{kg}$ and $100 \mu \mathrm{g} / \mathrm{kg}$ ) were injected for $15 \mathrm{~d}$. Hair samples were taken before (d 1) and after drug administration (d 16). Only the results for $\mathrm{d} 16$ are shown. Means \pm SEM $(n=8$ per group) are presented. Groups were statistically compared by 1-way ANOVA followed by SNK post hoc test when appropriate; $* P<$ 0.05 vs. control group; ${ }^{\$} P<0.05$ vs. $20 \mu \mathrm{g} / \mathrm{kg}$ group. stress states lasting only $9 \mathrm{~d}$ and that $\mathrm{HC}$ is perhaps more sensitive than changes in adrenal or thymus weight.

Chronic IMO and the present CUS procedure are severe stressful situations. We then wanted to study a chronic stressful situation of lower intensity than the previous one. Restraint is clearly of lower intensity than IMO (35), and it is an extensively used model. We found that $30 \mathrm{~min}$ restraint for $14 \mathrm{~d}$ increased $\mathrm{HC}$, although the increase was, as expected, of lower magnitude than that observed after IMO and CUS.

The preceding results clearly demonstrated that $\mathrm{HC}$ is a sensitive index of chronic stress. However, it is unclear the extent to which accumulation of corticosterone in hair saturates, thus diminishing its validity as a marker when using severe stressors and/or more prolonged periods of exposure. In fact, a saturation effect could explain the lack of differences between IMOch and CUS. To answer this question, we performed an experiment administering 2 doses of a long-acting ACTH preparation (tetracosactide), which slowly releases ACTH and can stimulate the adrenal for hours after its administration. This procedure has been previously described in the literature and results in strong increases in adrenal weight (49-51). Our results demonstrated dose-dependent reductions in body weight and food intake, as well as dose-dependent increases in adrenal weight and HC. The decreases in food intake and body weight gain observed with the highest dose of ACTH are in accordance with a previous report (51). In contrast, the lowest dose did not affect food intake but nevertheless

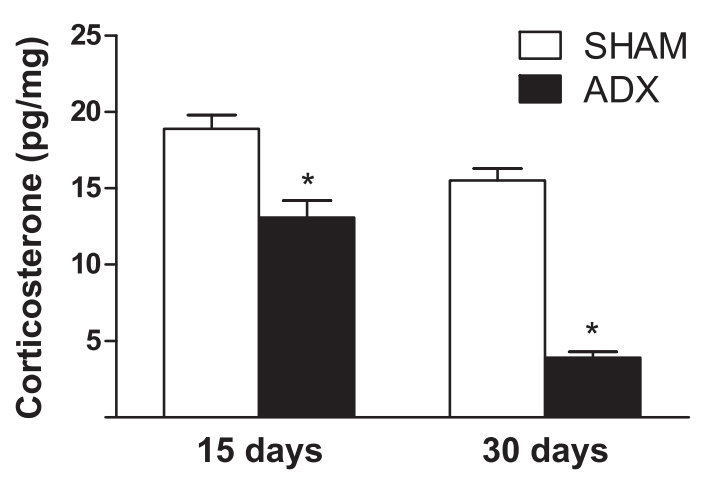

Figure 5. Effect of ADX on HC. Hair samples were collected at $\mathrm{d} 1$ (before surgery), 15, and 30, but only the results for d 15 and 30 are shown. Means $\pm \operatorname{SEM}(n=4$ per group) are presented. Groups were statistically compared by $t$ test; $* P<$ 0.05 vs. sham-treated group. 
decreased body weight, likely because of the well-known catabolic effects of glucocorticoids. Dramatic dosedependent increases in $\mathrm{HC}$ were observed after ACTH administration. These data not only confirm that HC reflects the magnitude of the chronic activation of the HPA axis but also show no evidence for saturation of corticosterone incorporation into the hair matrix. Therefore, it is clear that a ceiling effect in HC accumulation under more natural conditions of chronic stress is unlikely.

The preceding experiments indicate that $\mathrm{HC}$ is able to reflect increased in circulating corticosterone, but how this variable responds to reduced levels is unknown. Then we studied HC after ADX. In a pilot study, we observed only a partial decrease 2 weeks after ADX. Therefore, we repeated the experiment taking hair at 15 and $30 \mathrm{~d}$ after surgery. Again, we observed a moderate decrease after the first hair sampling, but after the second sampling, a much stronger decrease was found. It thus appears that hair shaved 2 wk after ADX only partially reflects the strong reduction of circulating corticosterone. One possible explanation is that the portion of hair under the skin, which accumulated corticosterone under regular conditions, erupted later to be sampled $2 \mathrm{wk}$ after ADX, thus partially masking the effect of ADX. However, it appears that this part of the hair takes at least $3 \mathrm{~d}$ to emerge (52), and it does not explain why the decrease in $\mathrm{HC}$ was so modest. Although the precise mechanisms of glucocorticoid incorporation to hair are unknown (19), it is possible that concentration of corticosterone is much higher in follicular cells that are generating the shaft than in the shaft itself. Therefore, it takes more days for the follicular cells born in absence of glucocorticoids to reflect the effect of ADX.

In conclusion, the washing and extraction procedure used was validated for rats and could also be potentially applied to other laboratory animals, such as mice. The present data indicate that $\mathrm{HC}$ concentration is similar in male and female rats and could reflect plasma-free corticosterone levels. HC was elevated in response to various chronic stressful procedures and chronic ACTH administration, demonstrating that it provides a good direct index of HPA activity over long periods of time that better compares with indirect parameters, such as adrenal or thymus weight. Although HC was eventually able to reflect the decrease in circulating corticosterone after ADX, this was clearly found only 3 to $4 \mathrm{wk}$ after the intervention. Considering the irregular hair growth of rats and the latter concern, it appears that this procedure could be most appropriate to evaluate chronic changes $(i . e ., 1 \mathrm{mo})$ in circulating corticosterone. With much shorter periods of time, classic urine and fecal samples taken several times are still valuable tools. Finally, this technique enables the use of rats to better characterize the impact of genetically or environmentally generated individual differences on the HPA axis and the impact on environmental conditions and chronic stressors, particularly those involving complex designs and stimuli.

This study was supported by grants from Marco Polo Program 2012, Ministerio de Ciencia e Innovación (SAF200801175, SAF2011-28313), Instituto de Salud Carlos III (RD20120028-0014, Redes Temáticas de Investigación Cooperativa en Salud, Ministerio de Sanidad y Consumo), and Generalitat de Catalunya (SGR2014-1020).

\section{REFERENCES}

1. Sapolsky, R. M., Romero, L. M., and Munck, A. U. (2000) How do glucocorticoids influence stress responses? Integrating permissive, suppressive, stimulatory, and preparative actions. Endocr. Rev. 21, 55-89

2. Armario, A. (2006) The hypothalamic-pituitary-adrenal axis: what can it tell us about stressors? CNS Neurol. Disord. Drug Targets 5, 485-501

3. McEwen, B. S. (1998) Stress, adaptation, and disease. Allostasis and allostatic load. Ann. N. Y. Acad. Sci. 840, 33-44

4. Armario, A., Daviu, N., Muñoz-Abellán, C., Rabasa, C., Fuentes, S., Belda, X., Gagliano, H., and Nadal, R. (2012) What can we know from pituitary-adrenal hormones about the nature and consequences of exposure to emotional stressors? Cell. Mol. Neurobiol. 32, 749-758

5. Hellhammer, J., Fries, E., Schweisthal, O. W., Schlotz, W., Stone, A. A., and Hagemann, D. (2007) Several daily measurements are necessary to reliably assess the cortisol rise after awakening: stateand trait components. Psychoneuroendocrinology 32, 80-86

6. Lightman, S. L., Wiles, C. C., Atkinson, H. C., Henley, D. E., Russell, G. M., Leendertz, J. A., McKenna, M. A., Spiga, F., Wood, S. A., and Conway-Campbell, B. L. (2008) The significance of glucocorticoid pulsatility. Eur. J. Pharmacol. 583, 255-262

7. Rooney, N. J., Gaines, S. A., and Bradshaw, J. W. S. (2007) Behavioural and glucocorticoid responses of dogs (Canis familiaris) to kennelling: investigating mitigation of stress by prior habituation. Physiol. Behav. 92, 847-854

8. Buchanan, K. L., and Goldsmith, A. R. (2007) Noninvasive endocrine data for behavioral studies: the importance of validation. Anim. Behav. 67, 183-185

9. Davenport, M. D., Tiefenbacher, S., Lutz, C. K., Novak, M. A., and Meyer, J. S. (2006) Analysis of endogenous cortisol concentrations in the hair of rhesus macaques. Gen. Comp. Endocrinol. 147, 255-261

10. Kintz, P. (2004) Value of hair analysis in postmortem toxicology. Forensic Sci. Int. 142, 127-134

11. Webb, E., Thomson, S., Nelson, A., White, C., Koren, G., Rieder, M., and Van Uum, S. H. M. (2010) Assessing individual systemic stress through cortisol analysis of archaeological hair. J. Archaeol. Sci. 37, 807-812

12. Yamada, J., Stevens, B., de Silva, N., Gibbins, S., Beyene, J., Taddio, A., Newman, C., and Koren, G. (2007) Hair cortisol as a potential biologic marker of chronic stress in hospitalized neonates. Neonatology 92, 42-49

13. Van Uum, S. H. M., Sauvé, B., Fraser, L. A., Morley-Forster, P., Paul, T. L., and Koren, G. (2008) Elevated content of cortisol in hair of patients with severe chronic pain: a novel biomarker for stress. Stress 11, 483-488

14. Dettenborn, L., Tietze, A., Bruckner, F., and Kirschbaum, C. (2010) Higher cortisol content in hair among long-term unemployed individuals compared to controls. Psychoneuroendocrinology 35, 1404-1409

15. Karlén, J., Ludvigsson, J., Frostell, A., Theodorsson, E., and Faresjö, T. (2011) Cortisol in hair measured in young adults-a biomarker of major life stressors? BMC Clin. Pathol. 11, 12

16. Grassi-Oliveira, R., Pezzi, J. C., Daruy-Filho, L., Viola, T. W., Francke, I. D., Leite, C. E., and Brietzke, E. (2012) Hair cortisol and stressful life events retrospective assessment in crack cocaine users. Am. J. Drug Alcohol Abuse 38, 535-538

17. Skoluda, N., Dettenborn, L., Stalder, T., and Kirschbaum, C. (2012) Elevated hair cortisol concentrations in endurance athletes. Psychoneuroendocrinology 37, 611-617

18. Fairbanks, L. A., Jorgensen, M. J., Bailey, J. N., Breidenthal, S. E., Grzywa, R., and Laudenslager, M. L. (2011) Heritability and genetic correlation of hair cortisol in vervet monkeys in low and higher stress environments. Psychoneuroendocrinology 36, 1201-1208

19. Meyer, J. S., and Novak, M. A. (2012) Hair cortisol: a novel biomarker of hypothalamic-pituitary-adrenocortical activity [minireview]. Endocrinology 153, 4120-4127

20. Russell, E., Koren, G., Rieder, M., and Van Uum, S. (2012) Hair cortisol as a biological marker of chronic stress: current status, future directions and unanswered questions. Psychoneuroendocrinology 37, 589-601

21. Hiroshige, T., Abe, K., Wada, S., and Kaneko, M. (1973) Sex difference in circadian periodicity of CRF activity in the rat hypothalamus. Neuroendocrinology 11, 306-320 
22. Atkinson, H. C., and Waddell, B. J. (1997) Circadian variation in basal plasma corticosterone and adrenocorticotropin in the rat: sexual dimorphism and changes across the estrous cycle. Endocrinology 138, 3842-3848

23. Gala, R. R., and Westphal, U. (1965) Corticosteroid-binding globulin in the rat: studies on the sex difference. Endocrinology 77, $841-851$

24. Droste, S. K., de Groote, L., Atkinson, H. C., Lightman, S. L. Reul, J. M. H. M., and Linthorst, A. C. E. (2008) Corticosterone levels in the brain show a distinct ultradian rhythm but a delayed response to forced swim stress. Endocrinology 149, 3244-3253

25. Droste, S. K., de Groote, L., Lightman, S. L., Reul, J. M. H. M. and Linthorst, A. C. E. (2009) The ultradian and circadian rhythms of free corticosterone in the brain are not affected by gender: an in vivo microdialysis study in Wistar rats. J. Neuroendocrinol. 21, 132-140

26. Pastor-Ciurana, J., Rabasa, C., Ortega-Sánchez, J. A., SanchísOllè, M., Gabriel-Salazar, M., Ginesta, M., Belda, X., Daviu, N., Nadal, R., and Armario, A. (2014) Prior exposure to repeated immobilization or chronic unpredictable stress protects from some negative sequels of an acute immobilization. Behav. Brain Res. 265, 155-162

27. Giralt, M., and Armario, A. (1989) Individual housing does not influence the adaptation of the pituitary-adrenal axis and other physiological variables to chronic stress in adult male rats. Physiol. Behav. 45, 477-481

28. Belda, X., Márquez, C., and Armario, A. (2004) Long-term effects of a single exposure to stress in adult rats on behavior and hypothalamic-pituitary-adrenal responsiveness: comparison of two outbred rat strains. Behav. Brain Res. 154, 399-408

29. Cone, E. J., and Joseph, R. E. (1996) The potential for bias in hair testing for drugs of abuse. In Drug Testing Hair (Kintz, P., ed.), pp. 69-93, CRC Press, Boca Raton

30. Raul, J. S., Cirimele, V., Ludes, B., and Kintz, P. (2004) Detection of physiological concentrations of cortisol and cortisone in human hair. Clin. Biochem. 37, 1105-1111

31. Pragst, F., and Balikova, M. A. (2006) State of the art in hair analysis for detection of drug and alcohol abuse. Clin. Chim. Acta 370, 17-49

32. García, A., Martí, O., Vallès, A., Dal-Zotto, S., and Armario, A. (2000) Recovery of the hypothalamic-pituitary-adrenal response to stress. Effect of stress intensity, stress duration and previous stress exposure. Neuroendocrinology 72, 114-125

33. Dal-Zotto, S., Martí, O., and Armario, A. (2000) Influence of single or repeated experience of rats with forced swimming on behavioural and physiological responses to the stressor. Behav. Brain Res. 114, 175-181

34. Ulrich-Lai, Y. M., Figueiredo, H. F., Ostrander, M. M., Choi, D. C., Engeland, W. C., and Herman, J. P. (2006) Chronic stress induces adrenal hyperplasia and hypertrophy in a subregionspecific manner. Am. J. Physiol. Endocrinol. Metab. 291, E965-E973

35. Campmany, L., Pol, O., and Armario, A. (1996) The effects of two chronic intermittent stressors on brain monoamines. Pharmacol. Biochem. Behav. 53, 517-523

36. Riondel, A. M., Rebuffat, P., Mazzochi, G., Nussdorfer, G. G., Gaillard, R. C., Bockhorn, L., Nussberger, J., Vallotton, M. B., and Muller, A. F. (1987) Long-term effects of ACTH combined with angiotensin II on steroidogenesis and adrenal zona glomerulosa morphology in the rat. Acta Endocrinol. (Copenh.) 114, 47-54

37. Zelena, D., Mergl, Z., Foldes, A., Kovács, K. J., Tóth, Z., and Makara, G. B. (2003) Role of hypothalamic inputs in maintaining pituitary-adrenal responsiveness in repeated restraint. Am. J. Physiol. Endocrinol. Metab. 285, E1110-E1117

38. Macbeth, B. J., Cattet, M. R. L., Stenhouse, G. B., Gibeau, M. L., and Janz, D. M. (2010) Hair cortisol concentration as a nonivasive measure of long-term stress in free-ranging grizzly bears (Ursus arctos): considerations with implications for other wildlife. Can.J. Zool. 88, 935-949

39. Bennett, A., and Hayssen, V. (2010) Measuring cortisol in hair and saliva from dogs: coat color and pigment differences. Domest. Anim. Endocrinol. 39, 171-180

40. Priestley, G. C. (1966) Rates and duration of hair growth in the albino rat. J. Anat. 100, 147-157

41. Manenschijn, L., Spijker, A. T., Koper, J. W., Jetten, A. M., Giltay, E. J., Haffmans, J., Hoencamp, E., and van Rossum, E. F. C. (2012) Long-term cortisol in bipolar disorder: associations with age of onset and psychiatric co-morbidity. Psychoneuroendocrinology 37, 1960-1968

42. O'Brien, K. M., Tronick, E. Z., and Moore, C. L. (2012) Relationship between hair cortisol and perceived chronic stress in a diverse sample. Stress Health 6, DOI: 10.1002/smi.2475

43. Stalder, T., and Kirschbaum, C. (2012) Analysis of cortisol in hair-state of the art and future directions. Brain Behav. Immun. 26, 1019-1029

44. King, J. A., Rosal, M. C., Ma, Y., Reed, G., Kelly, T. A., Stanek III, E. J., and Ockene, I. S. (2000) Sequence and seasonal effects of salivary cortisol. Behav. Med. 26, 67-73

45. Larsson, C. A., Gullberg, B., Råstam, L., and Lindblad, U. (2009) Salivary cortisol differs with age and sex and shows inverse associations with WHR in Swedish women: a cross-sectional study. BMC Endocr. Disord. 9, 16

46. Lederbogen, F., Kühner, C., Kirschbaum, C., Meisinger, C., Lammich, J., Holle, R., Krumm, B., von Lengerke, T., Wichmann, H. E., Deuschle, M., and Ladwig, K. H. (2010) Salivary cortisol in a middle-aged community sample: results from 990 men and women of the KORA-F3 Augsburg study. Eur. J. Endocrinol. 163, 443-451

47. Kudielka, B. M., Hellhammer, D. H., and Wüst, S. (2009) Why do we respond so differently? Reviewing determinants of human salivary cortisol responses to challenge. Psychoneuroendocrinology 34, 2-18

48. Staufenbiel, S. M., Penninx, B. W. J. H., Spijker, A. T., Elzinga, B. M., and van Rossum, E. F. C. (2013) Hair cortisol, stress exposure, and mental health in humans: a systematic review. Psychoneuroendocrinology 38, 1220-1235

49. Stark, E., Ács, Z., Makara, G. B., and Mihály, K. (1968) The hypophyseal-adrenocortical response to various different stressing procedures in ACTH-treated rats. Can. J. Physiol. Pharmacol. 46, 567-571

50. Alario, P., Gamallo, A., Beato, M. J., and Trancho, G. (1987) Body weight gain, food intake and adrenal development in chronic noise stressed rats. Physiol. Behav. 40, 29-32

51. Giralt, M., Garcia-Marquez, C., and Armario, A. (1987) Previous chronic ACTH administration does not protect against the effects of acute or chronic stress in male rats. Physiol. Behav. 40 , $165-170$

52. Liu, L. Y., Guo, D. S., Xin, X. Y., and Fang, J. (2008) Observation of a system of linear loops formed by re-growing hairs on rat skin. Anat. Rec. (Hoboken) 291, 858-868

Received for publication June 23, 2014. Accepted for publication October 14, 2014. 